\title{
Trellises of Molecular Oxygen on Anatase $\mathrm{TiO}_{2}(101)$
}

\author{
Christian Dette, ${ }^{\dagger, \# \odot ~ M i g u e l ~ A . ~ P e ́ r e z-O s o r i o, ~}{ }^{\ddagger}, \# \odot$ Shai Mangel, ${ }^{\dagger}$ Feliciano Giustino, ${ }^{\ddagger}{ }^{\circledR} \odot$ \\ Soon Jung Jung, ${ }^{*}+\oplus$ and Klaus Kern ${ }^{\dagger, \|}$ \\ ${ }^{\dagger}$ Max Planck Institute for Solid State Research, Heisenbergstrasse 1, Stuttgart 70569, Germany \\ ${ }^{\ddagger}$ Department of Materials, University of Oxford, Parks Road, Oxford OX1 3PH, United Kingdom \\ ${ }^{\S}$ Department of Materials Science and Engineering, Cornell University, Ithaca, New York 14853, United States \\ "Institute de Physique de la Matière Condensée, École Polytechnique Fédérale de Lausanne, Lausanne 1015, Switzerland
}

Supporting Information

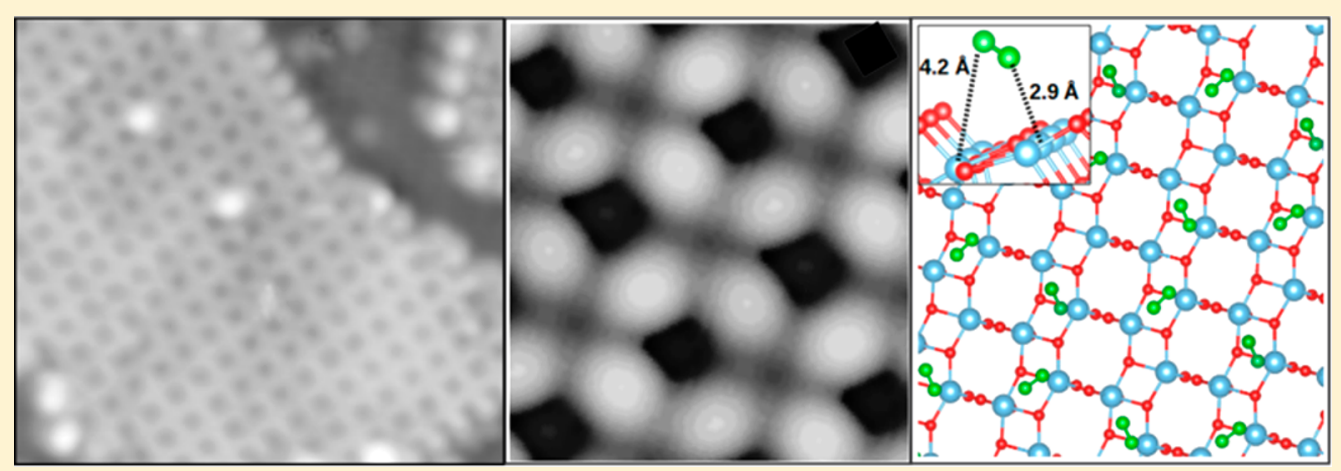

ABSTRACT: The adsorption of molecular oxygen has important implications for the catalytic activity of oxide surfaces, especially for oxidative $\mathrm{TiO}_{2}$ catalysts. In the case of anatase $\mathrm{TiO}_{2}(101)$, several studies have noted the importance of surface defects which stabilize the adsorption of oxygen molecules because of charge transfer. Further oxygen adsorption via this mechanism is precluded once a stoichiometric $\mathrm{TiO}_{2}$ surface is attained. Here, the adsorption of oxygen molecules was reenabled through a simple modification of sample cleaning procedures, resulting in the formation of a well-ordered trellis arrangement of oxygen molecules. The trellis is strongly passivating and does not affect the band gap of the pristine anatase substrate. Furthermore, they induce the extension of anatase terraces up to $100 \AA$ wide. The atomic and electronic structures of the trellis were investigated using scanning tunneling microscopy experimentation and density functional theory calculations.

\section{INTRODUCTION}

$\mathrm{TiO}_{2}$ is ubiquitous in environmental, energy, and biomedical applications because of its excellent structural, optical, and chemical properties. ${ }^{1-3}$ One of the most exciting aspects of $\mathrm{TiO}_{2}$ surface engineering is the ability to control the oxygen moieties on $\mathrm{TiO}_{2}$ because they significantly impact the chemical, optical, and electrical properties of the native oxide. ${ }^{4-7}$ On the stoichiometric $\mathrm{TiO}_{2}$ surface, the O:Ti ratio is 2; however, the actual ratio varies strongly with specific surface preparation methods. ${ }^{8-11}$ Cleaning procedures that entail sputtering and annealing in ultrahigh vacuum (UHV) typically result in oxygen-deficient surfaces with oxygen vacancies of variable concentration and distribution. Previously, we have shown that these oxygen vacancies can be assembled to cover large regions by increasing the temperature of the oxygen annealing step from 670 to $920 \mathrm{~K}$. $^{12}$ Moreover, the surface properties can be modified by an additional mechanism which involves oxygen vacancies diffusing into the subsurface owing to their low diffusion barriers, where they still remain as active centers for chemical reactivity. ${ }^{13,14}$ Besides studying these oxygen vacancies, the step edges are equally interesting because they are comprised entirely of assembled oxygen vacancies which are catalytically active. ${ }^{12}$

Additional to the oxygen vacancies, other atomic defects in $\mathrm{TiO}_{2}$ such as $\mathrm{Ti}$ interstitials and dopants introduce new properties to the $\mathrm{TiO}_{2}$ surface. The fundamental physical properties, electronic structure, and manipulation mechanisms of such defects, as well as their chemical activities, continue to be important subjects of research. ${ }^{15-18}$ Especially, molecular oxygen interacts with nearby oxygen vacancies through changing its oxidation state. ${ }^{14,19,20}$ It is activated at a 5-foldcoordinated surface $\mathrm{Ti}$ atom $\left(\mathrm{Ti}_{5 \mathrm{c}}\right)$ and adsorbs as either a singly charged superoxo $\left(\mathrm{O}_{2}^{-}\right)$or doubly charged peroxo $\left(\mathrm{O}_{2}{ }^{2-}\right)$, depending on the availability of excess electrons. ${ }^{14}$ The adsorption of neutral oxygen, however, is unknown because of its relatively low reactivity toward the stoichiometric anatase surface.

Received: May 7, 2019

Revised: September 27, 2019

Published: October 9, 2019 
In this article, we report on an unprecedented well-ordered network composed of neutral oxygen molecules on the anatase $\mathrm{TiO}_{2}(101)$ surface. All references to anatase in the foregoing text refer to this particular surface facet. Through simple modifications of a common surface-cleaning procedure, oxygen molecules were made to adsorb on anatase terraces and ordered into rhombic-shaped superstructures. These oxygen networks often expanded over step edges, producing new, exceptionally large terraces compared to those of the pristine anatase surface. Moreover, the oxygen network is chemically inert and does not introduce any gap states into the band gap of anatase.

\section{EXPERIMENTAL SECTION}

The experiments were carried out in a home-built $5 \mathrm{~K}$ scanning tunneling microscope (STM) using constant-current mode with a typical bias voltage of $1 \mathrm{~V}$ and a set point current of 0.05-1 nA. The STM tips were electrochemically etched Pt-Ir tips (N9801A) bought from Agilent Technologies, USA. The $\mathrm{d} I / \mathrm{d} V$ spectra were obtained using a lock-in amplifier modulating the bias voltage at a frequency of $2.5 \mathrm{kHz}$ above the cutoff frequency of the feedback loop with an amplitude of $20 \mathrm{mV}$.

We employed a naturally grown anatase $\mathrm{TiO}_{2}(101)$ single crystal which was polished to a surface roughness of less than one lattice constant (SurfaceNet $\mathrm{GmbH}$, Germany). The size of the crystal was $4 \times 4 \times 2 \mathrm{~mm}^{3}$ with a metallic, opaque color.

We prepared the crystal in situ in a preparation chamber (base pressure in the low $10^{-10} \mathrm{mbar}$ range) with two cycles of annealing in vacuum $(970 \mathrm{~K}, 30 \mathrm{~min})$ followed with $\mathrm{Ar}^{+}$sputtering ( $1 \mathrm{keV}$, sputtering current $2.2 \mu \mathrm{A}, 10 \mathrm{~min}$ ) and a postannealing step at a slightly reduced temperature $(920 \mathrm{~K}$, $30 \mathrm{~min}$ ). Afterward, we annealed the anatase sample at $670 \mathrm{~K}$ for 5 min followed by backfilling the chamber with oxygen $\left(670 \mathrm{~K}, 30 \mathrm{~min}, 1 \times 10^{-6} \mathrm{mbar}\right)$. In the last step we annealed the sample in a residual oxygen atmosphere $\left(\sim 3-6 \times 10^{-9}\right.$ mbar). The temperature ramp rate for heating was $2 \mathrm{~K} / \mathrm{s}$ while that for cooling was slower at $-0.5 \mathrm{~K} / \mathrm{s}$.

The oxygen network was prepared by reducing the temperature of the oxygen annealing from 670 to $620 \mathrm{~K}$ and increasing the duration of the postannealing from 30 to $60 \mathrm{~min}$ at $620 \mathrm{~K}$. The composition and the chemical state of surface constituents were determined by X-ray photoelectron spectroscopy (XPS) measurements (Figures S1-S3).

We complement our experiments with density functional theory (DFT) calculations, performed using the PerdewBurke-Ernzerhof exchange and correlation functional, ${ }^{21}$ as implemented in the Quantum ESPRESSO software package. ${ }^{22}$ Only valence electrons were explicitly described, including the Ti $3 s$ and $3 p$ semicore states. The core-valence interaction was taken into account through ultrasoft pseudopotentials. ${ }^{12}$ The wave functions and charge density were represented through plane-wave basis sets with energy cutoffs of 35 and $200 \mathrm{Ry}$, respectively. Spin polarization was included in our calculations. Surface Brillouin zone integrations were carried out using $2 \times 2$ Monkhorst-Pack meshes. The anatase surface was modeled with a slab containing four layers of $\mathrm{TiO}_{2}$. All structures were optimized with repeated conjugate-gradient minimizations until the forces on every atom were less than $0.04 \mathrm{eV} / \AA$. The STM images were calculated within the Tersoff-Hamann approximation at constant current, ${ }^{23}$ by considering all electronic states within $1 \mathrm{eV}$ from the conduction band edge.
We investigated the thermodynamic stability of the oxygen monolayer, in relation to the stability of the stoichiometric anatase (101) surface, and of an oxygen-deficient anatase (101) surface, by computing surface formation energies. These specific calculations were carried out with Hubbard corrections to the $\mathrm{Ti} 3 \mathrm{~d}$ states. While this allows us to correctly describe the surface state of the oxygen-deficient phase, it introduces a small penalty in the energetics of the $\mathrm{O}_{2}$ monolayer on $\mathrm{TiO}_{2}$ as we do not include Hubbard corrections to the $\mathrm{O} 2 \mathrm{p}$ states of $\mathrm{O}_{2}$ molecules. The Hubbard correction was included via a simplified rotationally invariant formulation. ${ }^{14} \mathrm{We}$ used a Hubbard parameter of $3.5 \mathrm{eV}$ ( Ti 3d). ${ }^{12}$ The formation energy, $\Delta E$, was calculated from the equation $\Delta E=1 / A\left(E_{\mathrm{slab}}-n \mu_{\mathrm{Ti}}\right.$ - $m \mu_{\mathrm{O}}$ ), subject to the following equilibrium conditions: (1) $\mu_{\mathrm{Ti}}+2 \mu_{\mathrm{O}}=E_{\mathrm{TiO}_{2} \text {,bulk }}$ and (2) $\mu_{\mathrm{O}}<{ }^{1} /{ }_{2} E_{\mathrm{O}_{2}}$. In these expressions, $E_{\text {slab }}$ is the total energy of the slab of the oxygen-deficient surface, stoichiometric surface, or oxygen monolayer; $A$ is the area of the surface unit cell; $\mu_{\mathrm{Ti}}$ and $\mu_{\mathrm{O}}$ are the chemical potentials of $\mathrm{Ti}$ and $\mathrm{O}$, respectively; $n$ and $m$ indicate the number of $\mathrm{Ti}$ and $\mathrm{O}$ atoms in the slab, respectively; $E_{\mathrm{TiO}_{2} \text {,bulk }}$ and $E_{\mathrm{O}_{2}}$ are the total energies per formula unit of bulk anatase and of an isolated $\mathrm{O}_{2}$ molecule, respectively. The thermodynamic relation between the oxygen chemical potential and the $\mathrm{O}_{2}$ partial pressure at a certain temperature is given by the following expression: $\mu_{\mathrm{O}}(T, P)=\mu_{\mathrm{O}}\left(T, P^{\mathrm{o}}\right)+{ }^{1} /{ }_{2} k T \ln \left(P / P^{\mathrm{o}}\right)$. In this equation, $T$ is the temperature, $P$ is the partial pressure of $\mathrm{O}_{2}, k$ is the Boltzmann constant, and $\mu_{\mathrm{O}}\left(T, P^{\circ}\right)$ is the oxygen chemical potential at standard conditions, that is, $P^{\circ}=1 \mathrm{~atm}$. The oxygen chemical potentials at standard conditions are taken from thermochemical tables. ${ }^{14}$

\section{RESULTS AND DISCUSSION}

Figure 1a is a STM image of a pristine anatase surface showing characteristic trapezoidal terraces with step edges oriented along the [010], $[11-1]$, and $[-111]$ directions. ${ }^{1}$ For the preparation of a pristine anatase surface, a combination of sputtering, annealing, and oxygen annealing is required as described above. By reduction of the temperature of oxygen annealing, a trellis-like structure is created on the anatase surface (Figure $1 \mathrm{~b}-\mathrm{d}$, detailed procedure in the Experimental Section). The network forms a superstructure with a rhombic unit cell of $7.4 \times 7.4 \AA^{2}$ and a small angle of $\alpha=83.5^{\circ}$ as revealed by Fourier-transforming (FT) the STM image of Figure $1 b$, shown in Figure 1c. The long diagonal of the rhombus is oriented along the [010] crystallographic direction of the anatase surface. Moreover, after the trellis structures are formed, large terraces of up to several hundred nanometers wide were observed (Figure 1b). These terrace widths are significantly larger than the typical $30 \mathrm{~nm}$ terrace widths found on the pristine anatase sample (Figure 1a).

A high-resolution STM topography of several patches of trellis on the anatase surface is shown in Figure 2. The isolated protrusions circled in green in Figure $2 b$ are frequently observed surrounding the trellis structure $(\sim 7 \AA)$, whereas almost no additional isolated protrusions are observed on top of the trellis structure, indicating that the trellis structure is chemically less reactive. The larger features at the network edge (blue arrows, $\sim 8 \AA$ ) are typically shifted $3.7 \AA$ from the lattice positions of the network (black dotted circle), which indicates that they are not bound tightly into the network. The smaller features at the network edge (yellow arrows, $\sim 6 \AA$ ) coincide with the lattice points of the trellis structure (yellow 


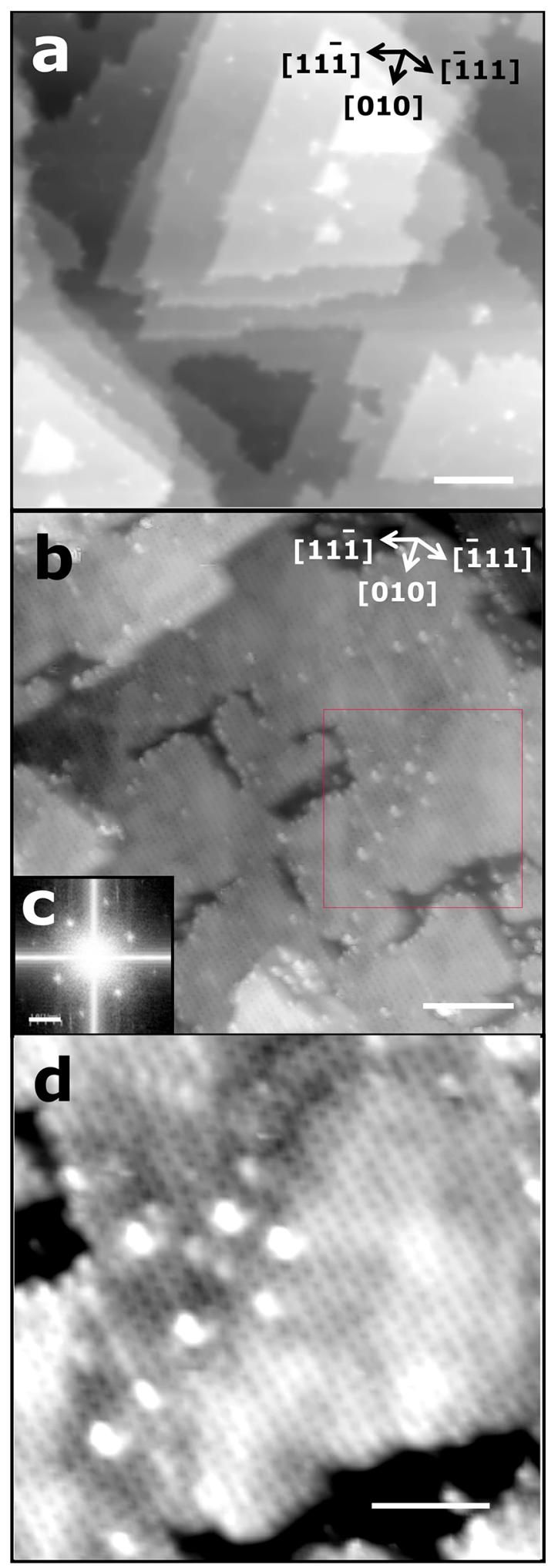

Figure 1. Topography of trellis structure grown on $\mathrm{TiO}_{2}$ anatase (101). (a) STM image of a pristine surface. (b) STM image reveals a large-scale trellis structure (local coverage up to $95 \%$ of a monolayer). (c) A Fourier transform of the STM image shows that the trellis forms a rhombic superstructure with a unit cell of $7.4 \times 7.4 \AA$ and a small angle of $\alpha=83.5^{\circ}$. (d) STM image enlarged from the red square in (b). (Scale bars: (a,b) $10 \mathrm{~nm},(\mathrm{c}) 1 \mathrm{~nm}$, and (d) $5 \mathrm{~nm}$.)

dotted circle). Some of the trellis extends until it reaches other trellis structures. We often found grain boundaries between two different network patches (marked with blue dotted lines in Figure 2d) that are connected but misaligned (marked with red dotted lines in Figure $2 \mathrm{~d}$ ) by the distance of exactly two neighboring $\mathrm{Ti}-\mathrm{O}$ moieties in the anatase (101) lattice. Moreover, a closer inspection of the trellis structures reveals that the border between the trellis structure and the step edges of anatase is not aligned along the crystallographic directions of pristine anatase surface (red arrows in Figure 2a,c). The modified step edges appear only when the trellis structures reach the anatase step edges, indicating that the extension of the trellis structure induces the modification of anatase surface. Figure 2e, (i) and (ii), shows differential conductance measurements performed on the network (the red circle in Figure 2a) which reveals a featureless large band gap identical to that of clean anatase (101) (measured at the black circle in Figure 2a).

The composition and the chemical state of trellis structure were studied by XPS experiment (Figures S1 and S2). The Ti $2 \mathrm{p}$ and $\mathrm{O} 1 \mathrm{~s}$ peaks in the XPS spectra suggest that the network consists solely of oxygen molecules. We also exclude surface impurities as the main cause for the observed structures because there was any change in impurity-related peaks before and after trellis structure formation.

To gain further insight into the trellis structure on the anatase (101) surface, a set of first-principles calculations were carried out. We first explored several atomistic models for a single oxygen molecule on anatase (101). In Figure 3a, the atomistic model with the lowest total energy is presented. We found that this model is slightly more stable than the model for a single oxygen molecule proposed by Setvin et al. ${ }^{14,20}$ by as much as $45 \mathrm{meV}$. Therefore, the model of Figure 3a is more likely to be the ground-state structure. For clarity, our model and the model proposed by Setvin et al. are compared in Figure S4. In our model (Figure 3a, Figure S4a) one of the oxygen atoms of the molecule sits atop a surface $\mathrm{Ti} 5 \mathrm{c}$ atom and the principle axis of the molecule is almost normal to the surface, while in Setvin's model the axis of the molecule is parallel to the surface (Figure S4b). We simulated the STM image of the single $\mathrm{O}_{2}$ molecule in the adsorption geometry of Figure 3a, and our result is presented in Figure 3b,c. As we can see, it is in excellent agreement with round-shaped protrusion in our experimental data (green circles in Figure 2b).

After having analyzed the adsorption structure of isolated oxygen molecule, we now turn our focus to the oxygen adsorbed on step edges. The pristine anatase surface has step edges oriented along the $[010],[11-1]$, and $[-111]$ directions. ${ }^{1}$ It is well-known that the $[-111]$ and $[11-1]$ step edges are highly reactive and possess many defects, even after several cleaning cycles (Figure S5). ${ }^{12,24}$ After the trellis structures are formed, the highly reactive step edges appear in stripelike contrast (white arrows in Figure 2b) and are adsorbates-free. Considering that the step edges along the $[-111]$ and $[11-1]$ direction of the pristine surface are covered with oxygen vacancies, ${ }^{12}$ we believe that excess oxygen species (which dissociatively adsorb during preparation ${ }^{14}$ ) fill the vacancies of the step edges. We calculated the oxygendecorated $[-111]$ step edge (Figure $3 \mathrm{~d}$ ). The additional oxygen atoms on the step edge lead to the stripelike contrast that exhibits round protrusions between the $\mathrm{Ti}-\mathrm{O}$ dimer rows (Figure $3 \mathrm{e}$ and red circles in Figure S6), which shows a close agreement with STM experiment (Figure 3f). We believe that the passivation of the chemically active step edges with oxygen will lead to an overall reduction of the surface reactivity. 


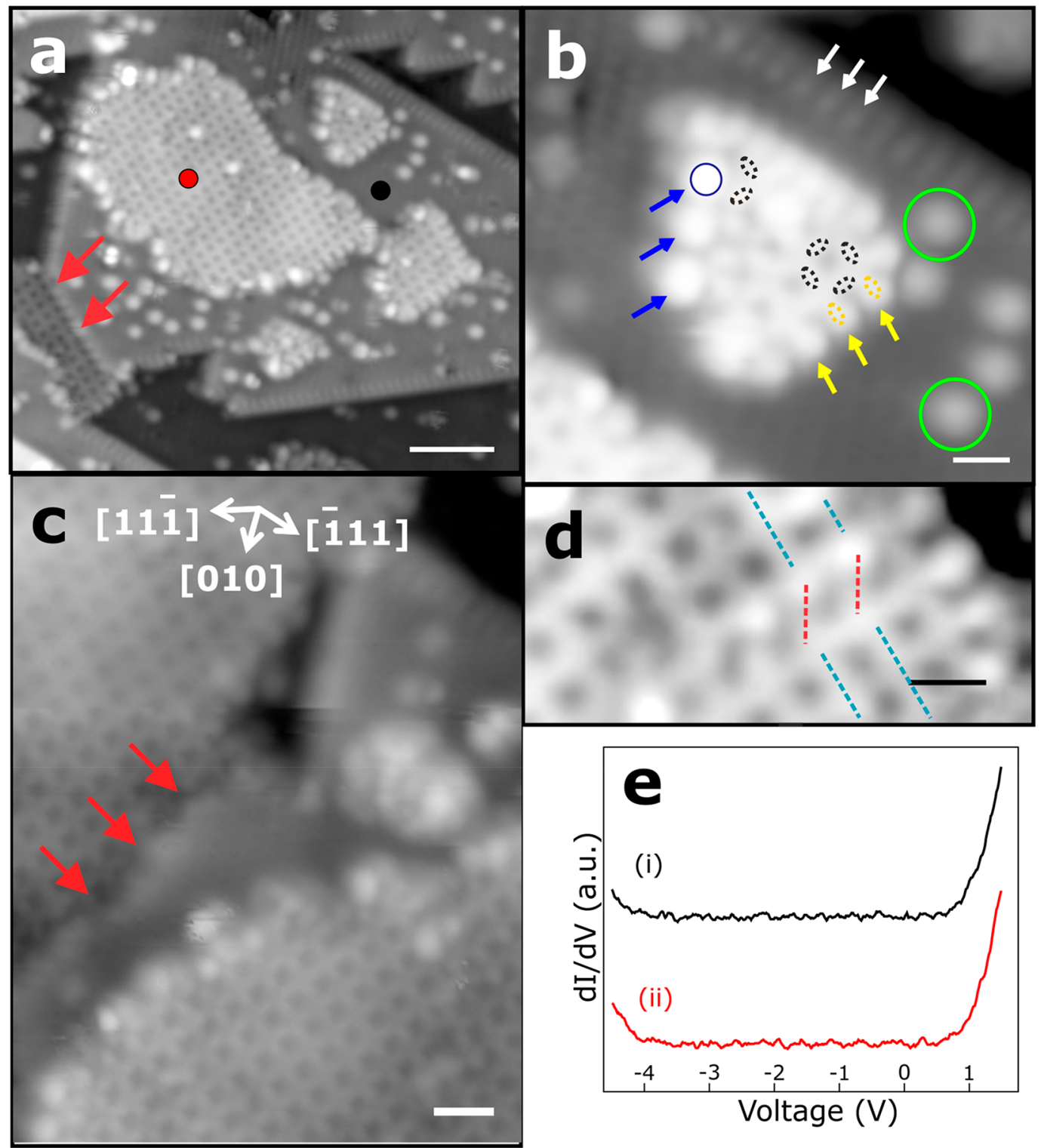

Figure 2. Higher resolution STM images of the trellis structures. (a) Trellis grown from a surface terrace. (b) Isolated protrusions are observed near the networks (green circles). We propose that, during sample preparation, the adsorbed species are mobile on the surface and can be incorporated into the network. This mechanism is supported by the presence of partially incorporated protrusions (blue and yellow arrows) near the network. The highly reactive step edges appear as stripelike structures (white arrows). (c) The directions of the step edges are not following those of the typical anatase (101) step edge configuration, indicative of the etching of the $\mathrm{TiO}_{2}$ surface. (d) A grain boundary occurring in larger network patches. (e) Tunneling spectroscopy obtained (i) on the pristine surface (black dot in (a)) and (ii) on top of the network (red dot in (a)) reveal the same featureless large band gap with no gap states. (Scale bars: $(\mathrm{a})=5 \mathrm{~nm},(\mathrm{~b})=1 \mathrm{~nm},(\mathrm{c})=2 \mathrm{~nm}$, and $(\mathrm{d})=1 \mathrm{~nm}$.)

Regarding the oxygen network, several structural candidates were considered, as shown in Figure S7. Among all the models considered, the most stable structure is presented in Figure 4a. The anatase (101) surface is composed of 2-fold coordinated oxygen $\left(\mathrm{O}_{2 \mathrm{c}}\right)$ and 5 -fold coordinated titanium $\left(\mathrm{Ti}_{5 \mathrm{c}}\right)$, while the bulk consists of 3-fold coordinated oxygen $\left(\mathrm{O}_{3 \mathrm{c}}\right)$ and 6-fold coordinated titanium $\left(\mathrm{Ti}_{6 \mathrm{c}}\right){ }^{1}$ Each adsorbed molecular oxygen is coordinated to two surface $\mathrm{Ti}$ atoms with one oxygen atom having a Ti-O distance of $2.9 \AA$ Ahile the other one has a TiO distance of $4.2 \AA$ (inset Figure $4 \mathrm{a}$ ), which is relatively long for a chemical bond. As presented in the ball-and-stick model in Figure $4 \mathrm{a}$, every third surface $\mathrm{Ti}_{5 \mathrm{c}}$ atom has no oxygen molecule adsorbed on it, resulting in a total surface $\mathrm{Ti}$ to adsorbed atomic $\mathrm{O}$ ratio of 3 to 2 (Figure S7b). Figure $4 \mathrm{~b}$ shows the calculated STM image of the ball-and-stick model overlaid with the anatase (101) lattice. The main contribution to the density of states (DOS) of the network comes from the oxygen molecule which is far away from the surface. The thick white lines denote the two-dimensional (2D) rhombic Bravais lattice that describes the periodicity of the oxygen network in the STM image with a unit cell of $7.7 \times 7.7 \AA^{2}$ and a small angle of $\alpha=84.9^{\circ}$. This agrees well with our experimentally obtained values for the network structure in Figure 1c. In the simulated STM image in Figure 4b, the network displays a reduced DOS at the individual sites of the rhombus and an enhanced DOS (brighter) between the intersections, whereas our STM image displays the homogeneously distributed DOS of the oxygen network without change in electronic contrast. However, experimental STM images with a functionalized tip in Figure S8b strongly resemble the calculated STM images 


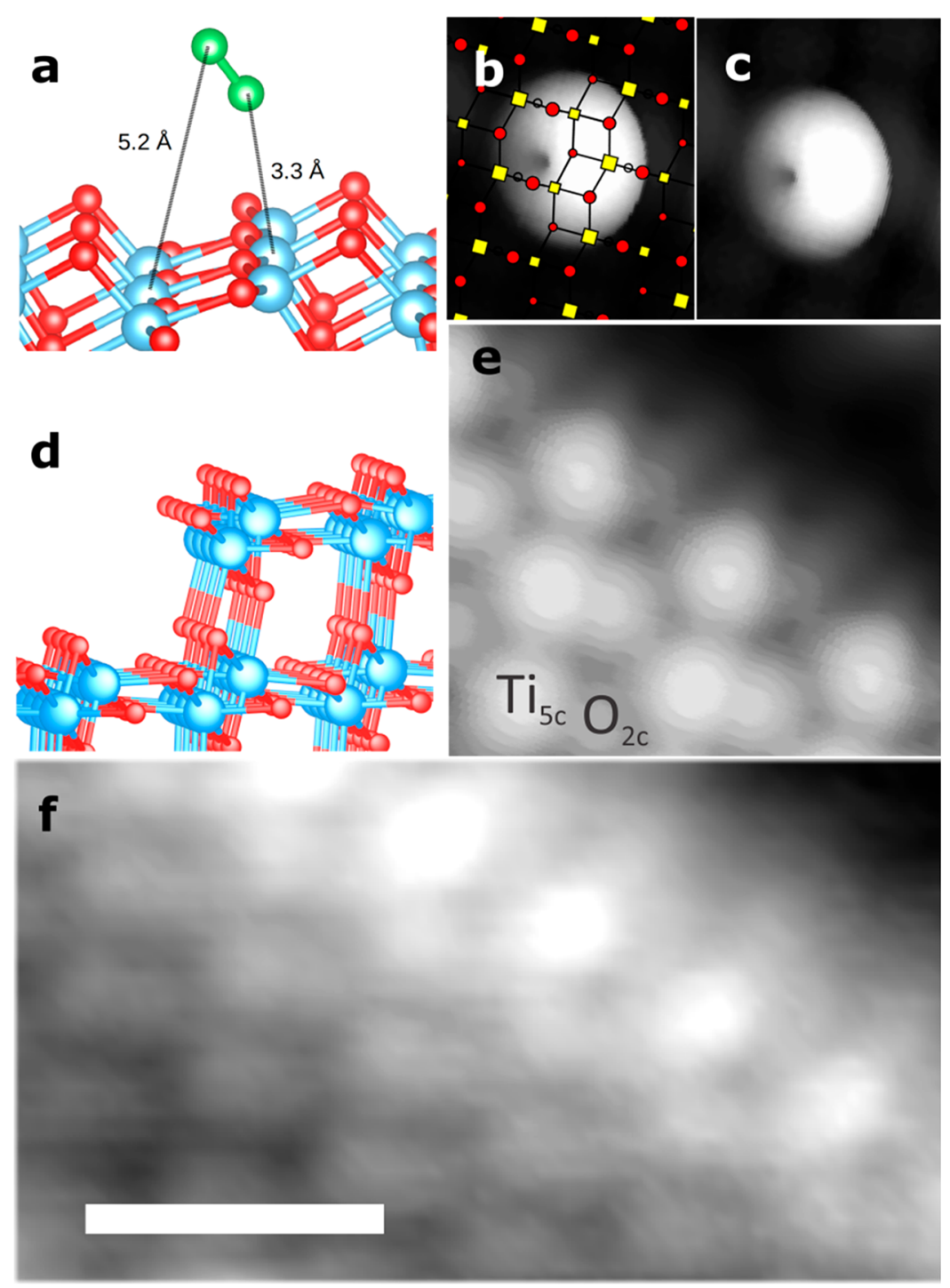

Figure 3. Calculated STM images of an oxygen molecule on the anatase (101) surface, and of the stoichiometric [-111] step edge. (a) Atomistic model of the single oxygen molecule on the anatase (101) surface and (b, c) its calculated empty-states STM image. In (b), the surface lattice is overlaid on the calculated STM image. (d) Atomistic model of the stoichiometric [-111] step edge on the anatase (101) surface and (e) its calculated STM image. In (a) and (d), the oxygen molecule is represented by green spheres, while the $\mathrm{O}$ and $\mathrm{Ti}$ atoms of the substrate are represented by red and blue spheres, respectively. The STM images are calculated by simulating constant current conditions by considering electronic states within $1.0 \mathrm{eV}(\mathrm{b}, \mathrm{c})$ and $1.1 \mathrm{eV}$ (e) from the conduction band edge. (f) Magnified experimental STM image (Vs $=2.0 \mathrm{~V}, I=0.1$ $\mathrm{nA})$ of the oxygen atoms adsorbed on the step edges oriented along the [-111] direction.

(Figure $4 \mathrm{~b}, \mathrm{c}$ ). We observed that the tip frequently became functionalized during scanning above the trellis structure, leading to higher scanning contrast that revealed further details in the electronic structure.

We calculated the electronic structure of the oxygen networks on anatase (101) in Figure $4 \mathrm{~d}$. The black curves in the differential conductance spectra resemble the projected DOS of the anatase substrate. It shows a large featureless band gap of $2 \mathrm{eV}$, which is in qualitative agreement with experiment, considering the band gap overestimation by tunneling spectroscopy $^{12}$ and underestimation by DFT-based calculations. ${ }^{13}$ The projected DOS of the oxygen network are depicted in red. The electronic feature of the network around $-0.5 \mathrm{~V}$ coincides with the onset of the valence band $(\mathrm{VB})$ of the substrate, while the feature around $+1.8 \mathrm{~V}$ coincides with the conduction band (CB) of the substrate. Hence, the calculated electronic structure of oxygen network on anatase explains the strong similarity between the differential conductance spectrum obtained on top of the substrate and on the network shown in Figure 2e. The calculated Löwdin charges of the single oxygen molecule (with a value of $5.94 \mathrm{e}$ on average) and of the $\mathrm{O}_{2}$ molecules ${ }^{4}$ forming the monolayer $(5.93 \mathrm{e})$ are very similar to those calculated for an isolated $\mathrm{O}_{2}$ molecule (5.95 e). Furthermore, in the single $\mathrm{O}_{2}$ molecule and $\mathrm{O}_{2}$ monolayer the calculated $\mathrm{O}-\mathrm{O}$ bond length $(1.21 \AA)$ matches closely to the experimental bond length of molecular $\mathrm{O}_{2}(1.2 \AA)$. These results further support the notion that the monolayer consists of electrically neutral $\mathrm{O}_{2}$ molecules.

We investigate the thermodynamic stability of the $\mathrm{O}_{2}$ monolayer on anatase (101), in relation to the stability of the pristine anatase (101) surface (abbreviated hereafter as Oterminated surface), and of the O-deficient anatase (101) surface (referred to as a Ti-terminated surface). The experimental observation of the Ti-terminated surface was 

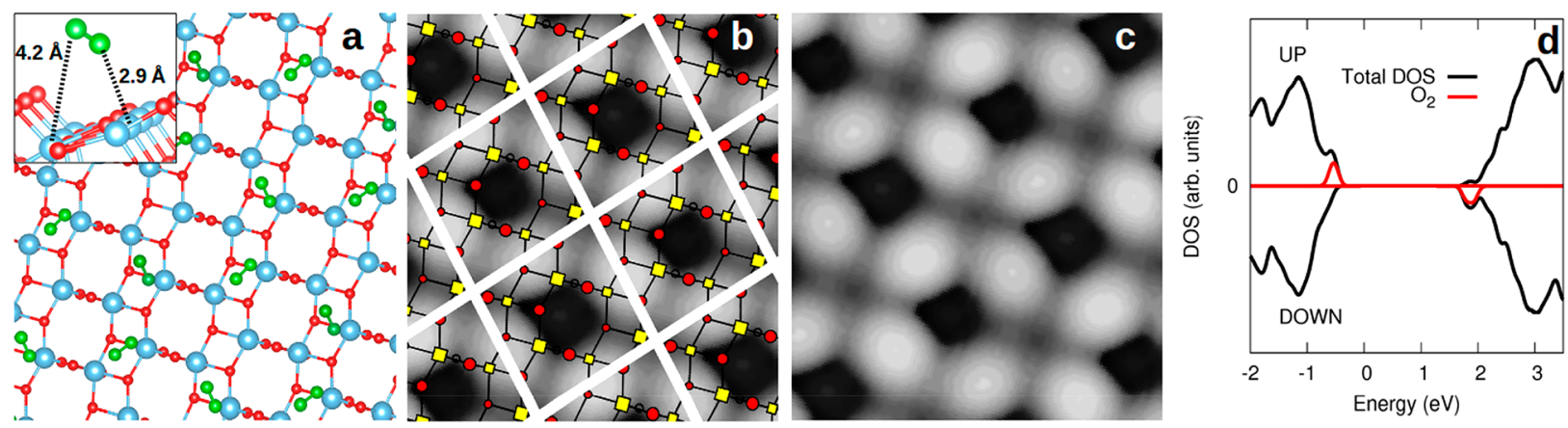

Figure 4. Atomistic model and calculated STM image of the oxygen monolayer on anatase (101). (a) Ball-and-stick model of the oxygen monolayer viewed from above, where molecular oxygen is represented with green spheres, and the $\mathrm{Ti}$ and $\mathrm{O}$ atoms of the substrate surface are indicated by blue and red spheres, respectively. The inset shows a cross-sectional view of one of the $\mathrm{O}_{2}$ molecules forming the monolayer. The Ti$\mathrm{O}$ distances of the adsorbed species are 2.9 and $4.2 \AA$, respectively. (b, c) The STM image of the oxygen monolayer was calculated at constant current mode by considering empty electronic states within $1.2 \mathrm{eV}$ from the bottom of the conduction band (isovalue $=1 \times 10^{-5} \mathrm{e} / \AA^{3}$ ) using the atomistic model shown in (a). In (b) the calculated STM image is overlaid with a schematic representation of the anatase (101) surface, where the large and small yellow rectangles represent surface $\mathrm{Ti}_{5 c}$ and $\mathrm{Ti}_{6 \mathrm{c}}$ atoms, respectively, and the large and small red circles stand for $\mathrm{O}_{2 c}$ and $\mathrm{O}_{3 c}$ atoms, respectively. The thick white lines denote the $2 \mathrm{D}$ rhombic Bravais lattice that describes the periodicity of the oxygen monolayer and the pattern of the STM image. The calculated parameters of the rhombic cell are $a=7.7 \AA$ and $\alpha=84.9^{\circ}$, which agree very well with the corresponding experimental values. (d) Calculated spin-resolved DOS for the oxygen monolayer on anatase (101). The total DOS is indicated by the black curve, while the red curve represents the partial DOS of the $\mathrm{O}_{2}$ molecules forming the monolayer. The Fermi energy is set to $0 \mathrm{eV}$. The DOS shows that the additional oxygen states of the monolayer are within the valence and conduction band (VB, $\mathrm{CB}$ ) of the underlying substrate, resulting in no additional band gap states.

reported in our previous work. ${ }^{12}$ The calculated formation energies of the Ti-terminated and O-terminated surfaces, and of the $\mathrm{O}_{2}$ monolayer, as a function of the oxygen chemical potential and $\mathrm{O}_{2}$ partial pressure, at 300,700 , and $900 \mathrm{~K}$, are shown in Figure S9. For the three temperatures considered here, the O-terminated surface is stable over a wide range of $\mathrm{O}_{2}$ partial pressures. Moreover, the stability window of the $\mathrm{O}$ terminated surface shifts to higher $\mathrm{O}_{2}$ partial pressures with increasing temperature. We can observe that the Ti-terminated surface becomes stable at high temperatures and $\mathrm{O}_{2}$ partial pressures close to UHV conditions. This observation is consistent with the experimental preparation conditions required to obtain a Ti-terminated surface. ${ }^{12}$ On the other hand, the oxygen monolayer is energetically favorable at higher $\mathrm{O}_{2}$ partial pressures compared to the Ti-terminated and $\mathrm{O}$ terminated surface. At the same $\mathrm{O}_{2}$ partial pressures, the formation energy of the oxygen monolayer increases as the temperature increases. This observation is also in line with the conditions used to prepare the oxygen monolayer in this work. Nevertheless, these theoretical results should be taken only as a qualitative description of the system because, as already pointed out previously, ${ }^{12}$ we found that the energetics of the O-terminated and Ti-terminated surfaces are sensitive to the parameters used in our calculations. Moreover, in the theoretical calculation, we did not consider the subsurface defects which may stabilize the adsorbed oxygen molecules.

Taken all together, the molecular oxygen favors being trapped either at a step edge or on a surface $\mathrm{Ti}_{5 \mathrm{c}}$ in the vicinity of subsurface extrinsic donor atoms along the [010] direction. ${ }^{14,20}$ We often observed step edges saturated with oxygen molecules even at very low oxygen coverage $(\sim 0.1$ ML). During the sample preparation at elevated temperatures, the oxygen molecules can diffuse and subsequently get incorporated into the network (blue arrows in Figure 2b). In our XPS spectra (Figures S1, S2, and S3), the peak position of the lattice oxygen $\left(E_{\mathrm{B}}=530.5 \mathrm{eV}\right)$ was preserved; that is, the binding energy shift due to the adsorption of charged oxygen molecules was not observed, which confirms that the majority of the adsorbed oxygen species are neutral. ${ }^{19}$ Additionally, the XPS peak indicating adsorbed atomic oxygen, which should appear at lower binding energy, was not observed. ${ }^{25,26}$ We also observed that the oxygen network is stable at room temperature.

At the step edges, the reorientation after oxygen adsorption is indicative of the oxygen-induced restructuring of the pristine anatase surface. The oxygen-induced surface restructuring on rutile $\mathrm{TiO}_{2}(110)$ was already reported. ${ }^{27-29}$ At typical oxygen annealing temperatures $(500-600 \mathrm{~K})$, the rutile crystal exhibits a diverse variety of surface restructurings that include formation of hexagonal rosettes, strands, and $(1 \times 1)$ islands. $^{27-29}$ These surface structures result from the oxidation of Ti interstitials that diffuse from the bulk to the surface. ${ }^{27-29}$ In contrast to the rutile, titanium interstitials and oxygen vacancies are not stable at the surface of anatase and, instead, are energetically favored to exist only in the subsurface region. ${ }^{14,30}$ Moreover, the reoriented step edges are existing only when the oxygen network reaches the step edge, which indicates that the oxygen network initiates the etching of the anatase surface at the step edges. However, the detailed mechanism of how the reconstruction proceeds between the oxygen network and the step edges will require further studies.

On the basis of the calculated adsorption energy of neutral oxygen molecules, the adsorption structure should not exist at $670 \mathrm{~K}$. However, previous research shows that subsurface defects stabilize adsorbed oxygen molecules irrespective of the exact nature of the charge donors in the subsurface region. ${ }^{14,19,20,31,32}$ Moreover, elevated temperatures enable the diffusion of these subsurface defects and any consequent reorientation of adsorbed oxygen molecules, which can bring about additional stabilization. ${ }^{14}$ Additional to the effect of the subsurface defect, our calculations (Figure S9) show that the trellis structure is energetically favorable over the pristine anatase surface, even at higher temperature, as long as the oxygen pressure is high enough. 


\section{CONCLUSIONS}

In summary, self-organization of oxygen molecules on the anatase (101) surface is studied using STM and DFT calculations. STM images reveal a well-organized rhombic superstructure with a unit cell size of $7.4 \times 7.4 \AA$. The molecular oxygen networks are chemically inert and do not affect the band gap of the pristine anatase substrate, hence making it suitable to serve as a surface-protecting layer. When we compare sample preperation procedures of the $\mathrm{O}$ terminated, ${ }^{12}$ Ti-covered, ${ }^{12}$ and oxygen-network-covered $\mathrm{TiO}_{2}$ anatase (101) surface, the difference is brought about by just modifying the oxygen annealing temperaure and $\mathrm{O}_{2}$ pressure. This comparison emphasizes the vast variation in surface morphology and surface chemistry accessible on the anatase (101) surface through simple modifications of the sample preparation conditions.

\section{ASSOCIATED CONTENT}

\section{S Supporting Information}

The Supporting Information is available free of charge on the ACS Publications website at DOI: 10.1021/acs.jpcc.9b04314.

XPS spectrum of oxygen network on anatase surface; calculations of the additional structural models and thermodynamic phase diagram (PDF)

\section{AUTHOR INFORMATION}

\section{Corresponding Author}

*E-mail: s.jung@fkf.mpg.de (S.J.J.).

\section{ORCID $\odot$}

Christian Dette: 0000-0002-4957-1029

Miguel A. Pérez-Osorio: 0000-0001-8077-5655

Feliciano Giustino: 0000-0001-9293-1176

Soon Jung Jung: 0000-0003-2428-5488

\section{Author Contributions}

${ }^{\#}$ C.D. and M.A.P.-O. contributed equally to this work. C.D. conducted the research with the help of S.M. and analyzed the data with S.J.J.; M.A.P.-O. performed the theoretical simulations under the supervision of F.G.; S.J.J. and K.K. supervised the project. All authors discussed the results and made critical contributions to the article.

\section{Notes}

The authors declare no competing financial interest.

\section{ACKNOWLEDGMENTS}

M.A.P.-O. and F.G. gratefully acknowledge funding from the Leverhulme Trust (Grant RL-2012-001), the UK Engineering and Physical Sciences Research Council (Grant No. EP/ M020517/1), and the Graphene Flagship (Horizon 2020 Grant No. 785219-GrapheneCore2); and supercomputing time at the University of Oxford Advanced Research Computing (ARC) facility (http://dx.doi.org/10.5281/ zenodo.22558).

\section{REFERENCES}

(1) Diebold, U. The surface science of titanium dioxide. Surf. Sci. Rep. 2003, 48 (5-8), 53-229.

(2) Linsebigler, A. L.; Lu, G.; Yates, J. T. Photocatalysis on $\mathrm{TiO}_{2}$ Surfaces: Principles, Mechanisms, and Selected Results. Chem. Rev. 1995, 95 (3), 735-758.
(3) Fei Yin, Z.; Wu, L.; Gui Yang, H.; Hua Su, Y. Recent progress in biomedical applications of titanium dioxide. Phys. Chem. Chem. Phys. 2013, 15 (14), 4844-4858.

(4) Iijima, K.; Goto, M.; Enomoto, S.; Kunugita, H.; Ema, K.; Tsukamoto, M.; Ichikawa, N.; Sakama, H. Influence of oxygen vacancies on optical properties of anatase $\mathrm{TiO}_{2}$ thin films. J. Lumin. 2008, 128 (5), 911-913.

(5) Buchalska, M.; Kobielusz, M.; Matuszek, A.; Pacia, M.; Wojtyła, S.; Macyk, W. On Oxygen Activation at Rutile- and Anatase- $\mathrm{TiO}_{2}$. ACS Catal. 2015, 5 (12), 7424-7431.

(6) Liu, G.; Yang, H. G.; Wang, X.; Cheng, L.; Lu, H.; Wang, L.; Lu, G. Q.; Cheng, H.-M. Enhanced Photoactivity of Oxygen-Deficient Anatase $\mathrm{TiO}_{2}$ Sheets with Dominant $\{001\}$ Facets. J. Phys. Chem. C 2009, 113 (52), 21784-21788.

(7) Etacheri, V.; Seery, M. K.; Hinder, S. J.; Pillai, S. C. Oxygen Rich Titania: A Dopant Free, High Temperature Stable, and Visible-Light Active Anatase Photocatalyst. Adv. Funct. Mater. 2011, 21 (19), 3744-3752.

(8) Göpel, W.; Anderson, J. A.; Frankel, D.; Jaehnig, M.; Phillips, K.; Schäfer, J. A.; Rocker, G. Surface defects of $\mathrm{TiO}_{2}(110)$ : A combined XPS, XAES AND ELS study. Surf. Sci. 1984, 139 (2), 333-346.

(9) Rusu, C. N.; Yates, J. T. Defect Sites on $\mathrm{TiO}_{2}(110)$. Detection by $\mathrm{O}_{2}$ Photodesorption. Langmuir 1997, 13 (16), 4311-4316.

(10) Pétigny, S.; Mostéfa-Sba, H.; Domenichini, B.; Lesniewska, E.; Steinbrunn, A.; Bourgeois, S. Superficial defects induced by argon and oxygen bombardments on (110) $\mathrm{TiO}_{2}$ surfaces. Surf. Sci. 1998, 410 (2), 250-257.

(11) Jackman, M. J.; Thomas, A. G.; Muryn, C. Photoelectron Spectroscopy Study of Stoichiometric and Reduced Anatase $\mathrm{TiO}_{2}(101)$ Surfaces: The Effect of Subsurface Defects on Water Adsorption at Near-Ambient Pressures. J. Phys. Chem. C 2015, 119 (24), 13682-13690.

(12) Dette, C.; Pérez-Osorio, M. A.; Kley, C. S.; Punke, P.; Patrick, C. E.; Jacobson, P.; Giustino, F.; Jung, S. J.; Kern, K. $\mathrm{TiO}_{2}$ Anatase with a Bandgap in the Visible Region. Nano Lett. 2014, 14 (11), 6533-6538.

(13) Cheng, H.; Selloni, A. Surface and subsurface oxygen vacancies in anatase $\mathrm{TiO}_{2}$ and differences with rutile. Phys. Rev. B: Condens. Matter Mater. Phys. 2009, 79 (9), 092101.

(14) Setvin, M.; Aschauer, U.; Scheiber, P.; Li, Y. F.; Hou, W.; Schmid, M.; Selloni, A.; Diebold, U. Reaction of $\mathrm{O}_{2}$ with Subsurface Oxygen Vacancies on $\mathrm{TiO}_{2}$ Anatase (101). Science 2013, 341 (6149), 988-991.

(15) Minato, T. Atomic Defects in Titanium Dioxide. Chem. Rec 2014, 14 (5), 923-934.

(16) Jupille, J.; Thornton, G. Defects at oxide surfaces; Springer: 2015; Vol. 58.

(17) Yin, W.-J.; Wen, B.; Zhou, C.; Selloni, A.; Liu, L.-M. Excess electrons in reduced rutile and anatase TiO2. Surf. Sci. Rep. 2018, 73 (2), 58-82.

(18) Dohnálek, Z.; Lyubinetsky, I.; Rousseau, R. Thermally-driven processes on rutile $\mathrm{TiO} 2(110)-(1 \times 1)$ : A direct view at the atomic scale. Prog. Surf. Sci. 2010, 85 (5), 161-205.

(19) Setvin, M.; Hulva, J.; Parkinson, G. S.; Schmid, M.; Diebold, U. Electron transfer between anatase $\mathrm{TiO}_{2}$ and an $\mathrm{O}_{2}$ molecule directly observed by atomic force microscopy. Proc. Natl. Acad. Sci. U. S. A. 2017, 114 (13), E2556-E2562.

(20) Setvin, M.; Daniel, B.; Aschauer, U.; Hou, W.; Li, Y.-F.; Schmid, M.; Selloni, A.; Diebold, U. Identification of adsorbed molecules via STM tip manipulation: $\mathrm{CO}, \mathrm{H}_{2} \mathrm{O}$, and $\mathrm{O}_{2}$ on $\mathrm{TiO}_{2}$ anatase (101). Phys. Chem. Chem. Phys. 2014, 16 (39), 21524-21530.

(21) Perdew, J.; Burke, K.; Ernzerhof, M. Generalized Gradient Approximation Made Simple. Phys. Rev. Lett. 1996, 77 (18), 38653868.

(22) Giannozzi, P.; Baroni, S.; Bonini, N.; Calandra, M.; Car, R.; Cavazzoni, C.; Ceresoli, D.; Chiarotti, G. L; Cococcioni, M.; Dabo, I.; Dal Corso, A.; de Gironcoli, S.; Fabris, S.; Fratesi, G.; Gebauer, R.; Gerstmann, U.; Gougoussis, C.; Kokalj, A.; Lazzeri, M.; MartinSamos, L.; Marzari, N.; Mauri, F.; Mazzarello, R.; Paolini, S.; 
Pasquarello, A.; Paulatto, L.; Sbraccia, C.; Scandolo, S.; Sclauzero, G.; Seitsonen, A. P; Smogunov, A.; Umari, P.; Wentzcovitch, R. M; et al. QUANTUM ESPRESSO: a modular and open-source software project for quantum simulations of materials. J. Phys.: Condens. Matter 2009, 21 (39), 395502.

(23) Tersoff, J.; Hamann, D. R. Theory of the scanning tunneling microscope. Phys. Rev. B: Condens. Matter Mater. Phys. 1985, 31 (2), 805-813.

(24) Dulub, O.; Diebold, U. Preparation of a pristine TiO2 anatase (101) surface by cleaving. J. Phys.: Condens. Matter 2010, 22 (8), 084014.

(25) Al-Ajlony, A.-M. B.; Kanjilal, A.; Harilal, S. S.; Hassanein, A. Carbon contamination and oxidation of $\mathrm{Au}$ surfaces under extreme ultraviolet radiation: An x-ray photoelectron spectroscopy study. J. Vac. Sci. Technol., B: Nanotechnol. Microelectron.: Mater., Process., Meas., Phenom. 2012, 30 (4), 041603.

(26) Ogasawara, H.; Naslund, L.; McNaughton, J.; Anniyev, T.; Nilsson, A. Double role of water in the fuel cell oxygen reduction reaction. ECS Trans. 2008, 16 (2), 1385-1394.

(27) Li, M.; Hebenstreit, W.; Diebold, U. Oxygen-induced restructuring of the rutile $\mathrm{TiO}_{2}(110)(1 \times 1)$ surface. Surf. Sci. 1998, 414 (1), L951-L956.

(28) Li, M.; Hebenstreit, W.; Diebold, U.; Henderson, M. A.; Jennison, D. R. Oxygen-induced restructuring of rutile $\mathrm{TiO}_{2}(110)$ : formation mechanism, atomic models, and influence on surface chemistry. Faraday Discuss. 1999, 114, 245-258.

(29) Onishi, H.; Iwasawa, Y. Dynamic Visualization of a MetalOxide-Surface/Gas-Phase Reaction: Time-Resolved Observation by Scanning Tunneling Microscopy at 800 K. Phys. Rev. Lett. 1996, 76 (5), 791-794.

(30) Cheng, H.; Selloni, A. Energetics and diffusion of intrinsic surface and subsurface defects on anatase $\mathrm{TiO}_{2}(101)$. J. Chem. Phys. 2009, 131 (5), 054703.

(31) Li, Y.-F.; Aschauer, U.; Chen, J.; Selloni, A. Adsorption and Reactions of $\mathrm{O} 2$ on Anatase $\mathrm{TiO} 2$. Acc. Chem. Res. 2014, 47 (11), 3361-3368.

(32) Aschauer, U.; Chen, J.; Selloni, A. Peroxide and superoxide states of adsorbed $\mathrm{O} 2$ on anatase $\mathrm{TiO} 2(101)$ with subsurface defects. Phys. Chem. Chem. Phys. 2010, 12 (40), 12956-12960. 